\title{
Post-Kala-Azar Dermal Leishmaniasis: A Paradigm of Paradoxical Immune Reconstitution Syndrome in Non-HIV/AIDS Patients
}

\author{
Eltahir Awad Gasim Khalil, ${ }^{1,2,3}$ Selma Abdelmoneim Khidir, ${ }^{1,2}$ \\ Ahmed Mudawi Musa, ${ }^{1,2}$ Brema Younis Musa, ${ }^{1,2}$ Mona Elfaki Eltahir Elfaki, ${ }^{1,2}$ \\ Abdelgadir Mohamed Yousif Elkadaru, ${ }^{1,4}$ Edward Zijlstra, ${ }^{1,2}$ \\ and Ahmed Mohamed El-Hassan ${ }^{1,2}$ \\ ${ }^{1}$ The Leishmaniasis Research Group, Sudan \\ ${ }^{2}$ Institute of Endemic Diseases, University of Khartoum, P.O. Box 45235, 11111 Khartoum, Sudan \\ ${ }^{3}$ The Central Laboratory, Ministry of Science \& Communications, 7099 Khartoum, Sudan \\ ${ }^{4}$ Tropical Diseases Hospital, Omdurman, Sudan \\ Correspondence should be addressed to Eltahir Awad Gasim Khalil; eltahirgasim@yahoo.ca
}

Received 22 November 2012; Accepted 21 February 2013

Academic Editor: Abul Faiz

Copyright (C) 2013 Eltahir Awad Gasim Khalil et al. This is an open access article distributed under the Creative Commons Attribution License, which permits unrestricted use, distribution, and reproduction in any medium, provided the original work is properly cited.

\begin{abstract}
Visceral leishmaniasis (VL) is a parasitic disease characterized by immune suppression. Successful treatment is usually followed by immune reconstitution and a dermatosis called post-Kala-azar dermal leishmaniasis (PKDL). Recently, PKDL was described as one of the immune reconstitution syndromes (IRISs) in HIV/VL patients on HAART. This study aimed to present PKDL as a typical example of paradoxical IRIS in non-HIV/AIDS individuals. Published and new data on the pathogenesis and healing of PKDL was reviewed and presented. The data suggested that PKDL is a typical example of paradoxical IRIS, being a new disease entity that follows VL successful treatment and immune recovery. PKDL lesions are immune inflammatory in nature with granuloma, adequate response to immunochemotherapy, and an ensuing hypersensitivity reaction, the leishmanin skin test (LST). The data also suggested that the cytokine patterns of PKDL pathogenesis and healing are probably as follows: an active disease state dominated by IL-10 followed by spontaneous/treatment-induced IL-12 priming, IL-2 stimulation, and INF- $\gamma$ production. INF- $\gamma$-activated macrophages eliminate the Leishmania parasites/antigen to be followed by LST conversion and healing. In conclusion, PKDL is a typical example of paradoxical IRIS in non-HIV/AIDS individuals with anti-inflammatory cytokine patterns that are superseded by treatmentinduced proinflammatory cytokines and lesions healing.
\end{abstract}

\section{Introduction}

L. donovani infections are widely prevalent in East Africa and the Indian subcontinent manifesting as a wide spectrum of clinical phenotypes ranging from subclinical infections to a potentially fatal visceral disease. Visceral leishmaniasis (VL) is a parasitic febrile illness with a transient immune suppression state with leucopenia and increased IL-10 secretion [1-4]. In the HIV/AIDS era, VL is considered an opportunistic infection as evidenced by emergence of
HIV/VL coinfections [5-10]. VL successful treatment is characterized by improvement of the leucopenia with a decline in CD4+ $\mathrm{T}$ cells and conversion in the leishmanin skin test (LST), a probable immunity surrogate marker. LST conversion probably indicates (re) constitution of transiently lost cell-mediated immunity against Leishmania antigens [1, 11-16]. In VL, IL-4 stimulation with IL-10 overproduction leads to reciprocal inhibition of INF- $\gamma$ production and polyclonal B-cells stimulation (Th2 immune response) [17-20]. 
More than fifty percent of successfully treated Sudanese VL patients develop an inflammatory skin rash, called postkala-azar dermal leishmaniasis (PKDL). A number of hypotheses have been put forward to explain the aetiology of PKDL: undertreatment, UVB light exposure, and ethnicity [1, 21-23]. LST conversion, high plasma and skin IL-10, high plasma levels of C-reactive protein and high TGF- $\beta$ during VL predict development, progression and severity of PKDL $[20,24,25]$. PBMCs and skin immune responses of VL/PKDL patients are dichotomous. It start as Th2 immune response in VL patients, pass through a mixed Th1/Th2 stage to be followed by a pure Th1 response in cured patients $[26,27]$. The majority of PKDL patients heal spontaneously, with persistence in $15 \%$ with chronic lesions that are probably a reservoir of infection $[22,28$, 29]. Sodium stibogluconate (SSG), liposomal amphotericin B (Ambisome), and immunochemotherapy (SSG in combination with alum-precipitated autoclaved $L$. major vaccine) are available treatment modalities [22, 30, 31].

Immune reconstitution inflammatory syndrome (IRIS) is a well-documented phenomenon that follows immune reconstitution in HIV patients who have recently started HAART. It is a stereotyped immune inflammatory state that is characterized by transient worsening or appearance of new symptoms/signs following successful treatment. IRIS has been described in patients with parasitic, bacterial, viral and autoimmune diseases [32-38]. CD4+ counts and preexisting opportunistic infection are reliable predictors of IRIS development [39-41]. The immune pathology of IRIS is largely determined by the infecting organisms where CD8+ $\mathrm{T}$ cells dominate lesions of viral origins; granulomatous inflammation usually dominates IRIS of fungi, protozoa, and mycobacterial conditions [42-50]. IRIS manifests when there is an abrupt shift from an anti-inflammatory and immunosuppressive state mediated by TNF- $\alpha$ and IL-10 to a proinflammatory state mediated by variable levels of IL-2, IL-12 and IFN- $\gamma$ [38, 51-55]. An increase in CD4+/CD 8+ cells coupled with a reduction in $\mathrm{T}_{\text {reg }}$ cells and an exaggerated cytokines response lead to initiation and progression of IRIS [56-61].

Recently, PKDL was reported as an IRIS phenomenon from Africa and Europe in HIV/AIDS/VL co-infected patients $[62,63]$.

This study aimed to present PKDL as a form of paradoxical IRIS in non-HIV/AIDS patients with plethora of cytokines production, granuloma formation, and delayedtype skin hypersensitivity reaction (LST) without activation of existing opportunistic infection.

\section{Materials and Methods}

Archived supernatant samples from in vitro stimulated PBMCs of thirty PKDL patients were selected from the samples bank of the Institute of Endemic Diseases, University of Khartoum. PKDL patients were enrolled in an immunochemotherapy study and were randomized to two study arms: patients in group I received four intradermal doses of $100 \mu \mathrm{g}$ alum-precipitated ALM + BCG (BCG 1/10th usual vaccine dose)/weekly plus daily sodium stibogluconate (SSG). Patients in group II received daily SSG plus four doses of the vaccine diluent (placebo). SSG was given intramuscularly/intravenously at a standard dose of $20 \mathrm{mg} / \mathrm{Kg}$ body weight/day [27].

The study protocol was reviewed and passed by the Ethics and Scientific Committees of the Institute of Endemic Diseases, University of Khartoum and the Ethics Committee of the Federal Ministry of Health, Khartoum. Samples were from patients who consented previously for the storage and use of their samples for further future testing. Patients were enrolled in the study based on specific inclusion and exclusion criteria as described previously [27].

PKDL patients were subjected to physical examination, pregnancy testing, DAT/HIV serological test, skin biopsy, haematological and chemical tests, LST, ECG, and cytokines at screening (D2), during treatment (D0, D7, D14, and D21) and follow up (D30, D40, D60, and D90) periods.

PBMCs were harvested using the density gradient centrifugation and were counted using Trypan blue exclusion technique with a haemocytometer. PBMCs cultures were stimulated with soluble $L$. donovani antigen; phytohemagglutinin (PHA) as a positive contol, a third well was left without antigen or mitogen as a negative control. Supernatants were stored at $-80^{\circ} \mathrm{C}$ for later analysis. IL-10 and IFN- $\gamma$ were measured using double sandwich technique as per manufacturer's leaf-lets (R\&D Systems, Germany). Results were previously reported [27]. IL-2 and IL-12 were measured using commercial kits (R\&D Systems, Germany).

\section{Results and Discussion}

3.1. Group I (Alum/ALM Vaccine/BCG + SSG). Total number of patients enrolled in this group was fifteen.

Five patients (data not shown on table) had low to undetectable IL-2, IL12, and IFN- $\gamma$ levels on D-2 (screening) and D60 in response to soluble $L$. donovani antigen (sLA). The leishmanin skin tests (LST) changed significantly from nonreactive (induration $=00 \mathrm{~mm}$ ) on $\mathrm{D}-2$ to reactive (induration $=8.0 \pm 1.4 \mathrm{~mm}$ ) on D60. All five patients healed completely by $\mathrm{D} 60$ of followup. These patients most probably passed IL-12 priming, IL-2 stimulation and IFN- $\gamma$ secretion (pro-inflammatory stage), and are now in leishmanin skin reactivity (memory) and lesion healing.

Four patients [nos. 107, 118, 116 and 129] showed low levels of IL- 2 and IL-12 on D-2 and D60. Their IFN- $\gamma$ was high on D2 but dropped significantly on D60 in response to soluble $L$. donovani antigen (sLA). Three $(3 / 4,75 \%)$ were LST reactive on D-2 and remained the same on D60. The fourth patient converted in LST on D60 of follow up. The skin lesions of the four patients healed completely by D60. It is probable that these patients passed IL-12 priming/IL-2 stimulation and were seen in IFN- $\gamma$ secretion (pro-inflammatory stage) where activated macrophages eliminated the Leishmania parasite/antigen paving the way for immune recovery (LST conversion) and healing (Table 1).

Three patients [nos. 121, 123 and 102] had low IL-2 and IL-12 on D- 2 and D60, the IFN- $\gamma$ levels increased significantly 
TABLE 1: IL-2, IL-12, INF- $\gamma$, and IL-10 levels and LST induration ( $\mathrm{mm}$ ) in some patients in the study.

\begin{tabular}{|c|c|c|c|c|c|c|c|c|c|c|c|}
\hline \multirow{2}{*}{ ID } & \multicolumn{5}{|c|}{ Day 2} & \multicolumn{5}{|c|}{ Day 60} & \multirow{2}{*}{ Treatment outcome } \\
\hline & IL-2 & IL-12 & INF- $\gamma$ & IL-10 & LST & IL-2 & IL-12 & INF- $\gamma$ & IL-10 & LST & \\
\hline $110^{*}$ & 716 & 00 & 2505 & 16 & 00 & 135 & 4.8 & 438 & 22 & 07 & Not healed \\
\hline $107^{*}$ & 55 & 2.9 & 2428 & 21 & 03 & 80 & 07 & 865 & 08 & 07 & Healed \\
\hline $104^{*}$ & 1220 & 4.8 & 2349 & 38 & 03 & 1530 & 18 & 1342 & 47 & 11 & Healed \\
\hline $116^{*}$ & 00 & 00 & 1016 & 33 & 08 & 34 & 03 & 968 & 81 & 10 & Healed \\
\hline $105^{*}$ & 150 & 00 & 720 & 00 & 00 & 30 & 18 & 27 & 48 & 06 & Healed \\
\hline $121^{*}$ & 00 & 08 & 00 & 00 & 00 & 20 & 04 & 531 & 22 & 07 & Healed \\
\hline $123^{*}$ & 00 & 00 & 101 & 04 & 00 & 00 & 06 & 209 & 09 & 10 & Healed \\
\hline $118^{*}$ & 00 & 02 & 427 & 12 & 08 & 00 & 4.8 & 85 & 36 & 09 & Healed \\
\hline $129^{*}$ & 00 & 00 & 342 & 19 & 06 & 15 & 16.5 & 270 & 40 & 11 & Healed \\
\hline $102^{*}$ & 00 & 1.8 & 74 & 00 & 00 & 42 & 4.8 & 874 & 35 & 07 & Healed \\
\hline 112 & 970 & 06 & 2584 & 345 & 03 & 45 & 00 & 151 & 08 & 08 & Healed \\
\hline 109 & 00 & 3.6 & 2584 & 09 & 00 & 25 & 05 & 626 & 17 & 08 & Healed \\
\hline 108 & 155 & 00 & 2271 & 19 & 00 & 185 & 00 & 1231 & 00 & 05 & Healed \\
\hline 106 & 45 & 4.8 & 2193 & 00 & 00 & 420 & 10.5 & 1342 & 29 & 00 & Healed \\
\hline 103 & 00 & 3.6 & 1732 & 00 & 00 & 52 & 10 & 1342 & 12 & 06 & Healed \\
\hline 101 & 25 & 18 & 1081 & 33 & 00 & 100 & 02 & 1342 & 32 & 00 & Not healed \\
\hline 130 & 00 & 1.2 & 813 & 102 & 04 & 00 & 00 & 14 & 20 & - & Not healed \\
\hline 115 & 00 & 00 & 118 & 113 & 08 & 23 & 18 & 704 & 62 & 12 & Healed \\
\hline 127 & 00 & 6.0 & 00 & 46 & 00 & 00 & 12.9 & 430 & 65 & 07 & Healed \\
\hline
\end{tabular}

${ }^{*}$ Group I patients (SSG + vaccine). IFN- $\gamma$, IL-2, Il-12, and IL-10 levels are expressed in pictogram $/ \mathrm{mL}$; leishmanin skin test (LST) induration is expressed in $\mathrm{mm}$.

on D60 compared to screening levels in response to soluble L. donovani antigen (sLA) and were accompanied by LST conversion. All three patients healed completely by D60. These patients already passed IL-12 priming/IL-2 stimulation when screened and were slowly creeping into IFN- $\gamma$ secretion (pro-inflammatory stage) and LST conversion and healing. Alternatively, these patients probably passed the IL-12 priming when screened and were not yet in the IL-2 stimulation. IL-2 stimulation was probably initiated later when immunechemotherapy was started, leading to increased IFN- $\gamma$ secretion and LST conversion and healing (Table 1).

Two patients [nos. 110, 105] had high IL-2 levels on D-2 that significantly drop on D60, while their IL-12 was low at both dates. Their IFN- $\gamma$ levels dropped significantly on D60 compared to their D-2 levels in response to soluble $L$. donovani antigen (sLA). Both patients converted in LST by D60 of follow up. One patient healed while the other did not and had to receive Ambisome treatment for the lesions to heal. These patients passed the IL-12 priming stage and were seen at the IL- 2 stimulation/IFN- $\gamma$ secretion stage (proinflammatory stage) that is followed by IFN $-\gamma$ reduction and LST conversion (Table 1).

One patient [nos. 104] had high IL-2 on D-2 that increased significantly on D60, while IL-12 was low through the follow up period. The IFN- $\gamma$ level was markedly high on D-2 and dropped significantly on D60 with LST conversion. This patient completely healed on D60 of follow up. This patient was probably in IL-2 stimulation/IFN- $\gamma$ secretion with overlap of LST conversion and healing (Table 1).
It is evident that all patients in the Alum/ALM vaccine + BCG group passed the anti-inflammatory stage when screened (low to absent IL-10), some were in the proinflammatory (IL-2/IL-12/IFN- $\gamma$ secretion) with progression to LST conversion and healing by D60. Their LST mean induration was $8.3 \pm 1.7 \mathrm{~mm}$ (median $=7 \mathrm{~mm}$ ) and was significantly different from D-2 LST induration $(P<0.000)$. Patients passed a probably short lived IL-12 priming state when screened. Some were in the IL-2 stimulation/IFN- $\gamma$ secretion/LST conversion/healing stage, while others had passed the IL-2 stimulation stage and were in IFN- $\gamma /$ LST conversion/healing. Majority of patients (14/15; 93.3\%) healed completely by D60. The SSG vaccine combination appears to be effective in eliminating the Leishmania parasite/antigen relieving the immune paresis that was preventing healing in these patients.

IL-10 levels were uniformly low in response to soluble L. donovani antigen (sLA) in all screening and follow up samples in all patients. This confirms that patients overcame the anti-inflammatory stage. The progression from an antiinflammatory to a pro-inflammatory stage has been reported previously as a prerequisite for the development of IRIS in HIV/TB co-infected patients on HAART [51-54].

3.2. Group II (SSG + Vaccine Diluent). Total number of patients enrolled in this arm was fifteen.

Seven patients [nos. 109, 106, 103, 101, 130, 115 and 127] had low IL-2 and low IL-12 on D-2 with similar levels on D60 except for one patient [no. 106] who showed an increase in 
IL-2, a drop in IFN- $\gamma$ with no LST conversion, and a complete healing by D60. Six patients of the above $[6 / 7,85.7 \%$; nos. 109 , $106,103,101,130$, and 115] had high IFN- $\gamma$ levels on D-2, that is, in a pro-inflammatory stage. Five patients [5/7, 71.4\%; nos. $109,106,103,101,130]$ showed marked to moderate drop in D2 IFN- $\gamma$ levels, while the other two $[2 / 7,28.6 \% ; 115,127]$ had a significant increase in IFN- $\gamma$ on D60, that is, progressive IFN- $\gamma$ secretion (anti-inflammatory stage). The majority of the seven patients [87.5\%] were LST non-reactive on D-2 compared to $62.5 \%$ on D60. The majority $(85.7 \%)$ of these patients healed completely by D60. It is probable that most of these patients passed the IL12 priming (?Transient IL-12 priming) and the IL-2 stimulation and were in the IFN- $\gamma$ secretion stage when seen on D-2. On D60, some of these patients continued in IFN- $\gamma$ secretion, while others dropped their IFN- $\gamma$ with consequent LST conversion and healing (Table 1).

Six patients had low to undetectable IL-2, IL-12, and IFN- $\gamma$ in response to soluble $L$. donovani antigen (sLA) on D-2with similar levels on D60. The majority (5/6; 83\%) were LST non-reactive on D-2 and remained the same on D60 with only one healed patient. Another patient $(1 / 6 ; 17 \%)$ who was strongly LST reactive on D-2 and remained the same on D60; he progressed to complete healing. The healed two patients probably passed the IL-12 priming, the IL-2 stimulation and IFN- $\gamma$ production and were in LST conversion status with complete cure. The nonhealing 4 patients were probably in an "immune paresis" state and were not able to mount IL-12 priming, IL-2, and IFN- $\gamma$ production/LST conversion and so exhibited no healing (?high leishmania antigen load). These patients healed completely with Ambisome treatment. Failure to progress to IL-2 priming and IL-12/IFN- $\gamma$ secretion in these patients probably indicates a degree of parasite unresponsiveness to SSG leading to persistence of Leishmania parasite/antigen and the observed immune paresis as evidenced by lack of LST conversion. Ambisome was successful in overcoming the parasite unresponsiveness, which is indicated by lesions healing.

Patient no. 112 had low IL-12, high IL-2, and high IFN- $\gamma$ and LST non-reactivity on D-2. On D60, the IFN- $\gamma$ and IL-2 drop was accompanied by LST conversion and healing. This patient probably passed IL-12 priming and was in IL-2 stimulation and IFN- $\gamma$ secretion when screened. The IFN- $\gamma$ drop, LST conversion and healing were achieved during the follow up period. This patient findings demonstrate the mirror-image pattern exhibited by IL- 2 and IFN- $\gamma$ levels.

Patient no. 108 had low IL-12, high IL2/IFN- $\gamma$ on D2, with IL-2 remaining high on D60 with a drop in IFN- $\gamma$ level LST conversion and healing. The high level of IL- 2 with low IFN- $\gamma$ levels and LST conversion and healing needs explanation!

IL-10 levels in response to soluble $L$. donovani antigen (sLA) were uniformly low in all screening and follow up samples, that is, patients passed the anti-inflammatory state when screened. These patients overcame the anti-inflammatory stage, progressed to a pro-inflammatory in line with previous data on IRIS in HIV co-infected patients on HAART [51-54].

Some of the patients in the SSG/vaccine diluent were in a state of immune paresis (high leishmania antigen load) and were unable to mount an IL-12 priming and IL-2/IFN- $\gamma$
secretion/LST conversion and healing. As suggested previously, a state of parasite SSG-unresponsiveness could be a contributory factor in patients lingering in an immune paresis stage. Ambisome treatment eliminated the parasite with reduction in Leishmania antigenic load putting patients on the way to recovery. Others in this group showed the typical natural history of PKDL healing, that is, passing IL-12 priming and were seen in the IL-2/IFN- $\gamma$ secretion that was followed by LST conversion and healing.

Data from this study showed the clear dichotomy of immune response in PKDL patients as was previously reported [64]. On the other hand, the levels of IL-2 and IFN- $\gamma$ more or less mirror-image each other.

Case reports from Africa and Europe introduced PKDL as an immune reconstitution phenomenon in HIV/VL Coinfected patients at the start of HAART $[62,63]$. In this study we attempted to prove that PKDL is an IRIS phenomenon that develops in VL patients who go through a transient stage of immune depression. PKDL develops as a new disease entity with symptoms and signs that are mainly of skin origin and that are different from VL. Cured VL patients become immune competent as evidenced by conversion of the LST at six months after treatment, around the same time of PKDL development [29]. Healing of PKDL lesions is a function of a change of immune responses from a mixed Th1/Th2 state (anti-inflammatory) to a pure Th1 one (proinflammatory). Healing of skin lesions starts at the cellular level by antigen presentation, followed by IL-12 priming, and IL-2 secretion which facilitates expansion of the Th1 population and IFN- $\gamma$ and TNF- $\beta$ secretion. It is logical to assume that the sequence of events in PKDL healing is as follows: drug-induced parasite killing, antigen load reduction, IL-12 priming followed by IL-2 secretion that in turn induces IFN- $\gamma$ secretion which augments the killing potential of the macrophages with production of nitric oxide and reactive oxygen intermediates. Eventually, inflammation decreases and healing occurs with production of memory cells and a lifelong LST reactivity status. The data also points to the fact that the cytokine patterns of PKDL healing are stereotyped and the differences between drug-treated (SSG; Ambisome), and immunochemotherapy-induced patterns are only quantitative. IL-2 is most probably the initiating cytokine for the healing process in PKDL, a finding that may have future therapeutic implications.

In conclusion, PKDL is a form of paradoxical IRIS that emerges as a new disease entity following successful VL treatment and immune recovery. PKDL skin lesions are immune inflammatory in nature and respond adequately to immuno-chemotherapy. Like IRIS, PKDL is an immunemediated phenomenon with increased activation from antigenic exposure, granuloma formation, and skin hypersensitivity reaction (LST conversion). Different Th1 and Th2 cytokines play important roles in PKDL pathogenesis and healing. IL-2 plays a pivotal role in PKDL healing process.

\section{Conflict of Interests}

The authors declared that they have no conflict of interests. 


\section{Acknowledgments}

The authors would like to express their thanks and gratitude to the staff of the Tropical Diseases Hospital, Omdurman, for their considerable help. The team received financial support from the Institute of Endemic Diseases, University of Khartoum.

\section{References}

[1] E. E. Zijlstra and A. M. El Hassan, "Leishmaniasis in Sudan. Visceral leishmaniasis," Transactions of the Royal Society of Tropical Medicine and Hygiene, vol. 95, pp. S27-S58, 2001.

[2] S. L. Croft, S. Sundar, and A. H. Fairlamb, "Drug resistance in leishmaniasis," Clinical Microbiology Reviews, vol. 19, no. 1, pp. 111-126, 2006.

[3] A. Hailu, A. Musa, M. Wasunna et al., "Geographical variation in the response of visceral leishmaniasis to paromomycin in East Africa: a multicentre, open-label, randomized trial," PLoS Neglected Tropical Diseases, vol. 4, no. 10, article e709, 2010.

[4] P. K. Sinha, P. Roddy, P. P. Palma et al., "Effectiveness and safety of liposomal amphotericin b for visceral leishmaniasis under routine program conditions in Bihar, India," American Journal of Tropical Medicine and Hygiene, vol. 83, no. 2, pp. 357-364, 2010.

[5] P. Desjeux, "Leishmania/HIV co-infections," Africa Health, vol. 18, no. 1, pp. 20-22, 1995.

[6] P. Mathur, J. C. Samantaray, M. Vajpayee, and P. Samanta, "Visceral leishmaniasis/human immunodeficiency virus coinfection in India: the focus of two epidemics," Journal of Medical Microbiology, vol. 55, part 7, pp. 919-922, 2006.

[7] N. Ezra, M. T. Ochoa, and N. Craft, "Human immunodeficiency virus and leishmaniasis," Journal of Global Infectious Diseases, vol. 2, no. 3, pp. 248-257, 2010.

[8] Z. Hurissa, S. Gebre-Silassie, W. Hailu et al., "Clinical characteristics and treatment outcome of patients with visceral leishmaniasis and HIV co-infection in northwest Ethiopia," Tropical Medicine and International Health, vol. 15, no. 7, pp. 848-855, 2010.

[9] J. Zhou, T. Sirisanthana, S. Kiertiburanakul et al., "Trends in CD4 counts in HIV-infected patients with HIV viral load monitoring while on combination antiretroviral treatment: results from The TREAT Asia HIV Observational Database," BMC Infectious Diseases, vol. 10, article 361, 2010.

[10] E. T. Nascimento, M. L. N. Moura, J. W. Queiroz et al., "The emergence of concurrent HIV-1/AIDS and visceral leishmaniasis in Northeast Brazil," Transactions of the Royal Society of Tropical Medicine and Hygiene, vol. 105, no. 5, pp. 298-300, 2011.

[11] E. M. Carvalho, R. S. Teixeira, and W. D. Johnson, "Cellmediated immunity in American visceral leishmaniasis: reversible immunosuppression during acute infection," Infection and Immunity, vol. 33, no. 2, pp. 498-502, 1981.

[12] J. P. Haldar, S. Ghose, K. C. Saha, and A. C. Ghose, "Cellmediated immune response in Indian kala azar and post-kala azar dermal leishmaniasis," Infection and Immunity, vol. 42, no. 2, pp. 702-707, 1983.

[13] E. M. Carvalho, R. Badaro, S. G. Reed, T. C. Jones, and W. D. Johnson, "Absence of gamma interferon and interleukin 2 production during active visceral leishmaniasis," Journal of Clinical Investigation, vol. 76, no. 6, pp. 2066-2069, 1985.
[14] K. A. Weigle, L. Valderrama, A. L. Arias, C. Santrich, and N. G. Saravia, "Leishmanin skin test standardization and evaluation of safety, dose, storage, longevity of reaction and sensitization," American Journal of Tropical Medicine and Hygiene, vol. 44, no. 3, pp. 260-271, 1991.

[15] E. E. Zijlstra and A. M. El Hassan, "Leishmanin and tuberculin sensitivity in leishmaniasis in the Sudan, with special reference to kala-azar," Transactions of the Royal Society of Tropical Medicine and Hygiene, vol. 87, no. 4, pp. 425-427, 1993.

[16] E. A. G. Khalil, A. M. El Hassan, E. E. Zijlstra et al., "Autoclaved Leishmania major vaccine for prevention of visceral leishmaniasis: a randomised, double-blind, BCG-controlled trial in Sudan," The Lancet, vol. 356, no. 9241, pp. 1565-1569, 2000.

[17] A. E. Harith, A. H. J. Kolk, P. A. Kager et al., "Evaluation of a newly developed direct agglutination test (DAT) for serodiagnosis and sero-epidemiological studies of visceral leishmaniasis: comparison with IFAT and ELISA," Transactions of the Royal Society of Tropical Medicine and Hygiene, vol. 81, no. 4, pp. 603-606, 1987.

[18] H. W. Ghalib, M. R. Piuvezam, Y. A. W. Skeiky et al., "Interleukin 10 production correlates with pathology in human Leishmania donovani infections," Journal of Clinical Investigation, vol. 92, no. 1, pp. 324-329, 1993.

[19] S. Nylén and D. Sacks, "Interleukin-10 and the pathogenesisof human visceral leishmaniasis," Trends in Immunology, vol. 28, pp. 378-382, 2007.

[20] S. Saha, S. Mondal, R. Ravindran et al., "IL-10- and TGF- $\beta$ mediated susceptibility in kala-azar and post-kala-azar dermal leishmaniasis: the significance of amphotericin B in the control of Leishmania donovani infection in India," Journal of Immunology, vol. 179, no. 8, pp. 5592-5603, 2007.

[21] A. Ismail, Immune responses and immunopathology of post kala-azar dermal Leishmaniasis [Ph.D. thesis], University of Copenhagen, Copenhagen, Denmark, 1999.

[22] E. E. Zijlstra, A. M. Musa, E. A. G. Khalil, I. M. El Hassan, and A. M. El Hassan, "Post-kala-azar dermal leishmaniasis," The Lancet Infectious Diseases, vol. 3, no. 2, pp. 87-98, 2003.

[23] A. Ismail, E. A. G. Khalil, A. M. Musa et al., "The pathogenesis of post kala-azar dermal leishmaniasis from the field to the molecule: does ultraviolet light (UVB) radiation play a role?" Medical Hypotheses, vol. 66, no. 5, pp. 993-999, 2006.

[24] S. Gasim, A. M. El Hassan, E. A. G. Khalil et al., "High levels of plasma IL-10 and expression of IL-10 by keratinocytes during visceral leishmaniasis predict subsequent development of postkala-azar dermal leishmaniasis," Clinical and Experimental Immunology, vol. 111, pp. 64-69, 1998, Erratum in: Clinical and Experimental Immunology, vol. 112, pp. 574, 1998.

[25] S. Gasim, T. G. Theander, and A. M. El Hassan, "High levels of C-reactive protein in the peripheral blood during visceral leishmaniasis predict subsequent development of post kala-azar dermal leishmaniasis," Acta Tropica, vol. 75, no. 1, pp. 35-38, 2000.

[26] A. A. Kamil, E. A. G. Khalil, A. M. Musa et al., "Alumprecipitated autoclaved Leishmania major plus bacille Calmette-Guérrin, a candidate vaccine for visceral leishmaniasis: safety, skin-delayed type hypersensitivity response and dose finding in healthy volunteers," Transactions of the Royal Society of Tropical Medicine and Hygiene, vol. 97, no. 3, pp. 365-368, 2003.

[27] A. M. Musa, E. A. G. Khalil, F. A. E. Mahgoub et al., "Immunochemotherapy of persistent post-kala-azar dermal leishmaniasis: a novel approach to treatment," Transactions of 
the Royal Society of Tropical Medicine and Hygiene, vol. 102, no. 1, pp. 58-63, 2008.

[28] A. M. El Hassan and E. A. G. Khalil, "Post-kala-azar dermal leishmaniasis: does it play a role in the transmission of Leishmania donovani in the Sudan?" Tropical Medicine and International Health, vol. 6, no. 9, pp. 743-744, 2001.

[29] A. M. Musa, E. A. G. Khalil, M. A. Raheem et al., "The natural history of Sudanese post-kala-azar dermal leishmaniasis: clinical, immunological and prognostic features," Annals of Tropical Medicine and Parasitology, vol. 96, no. 8, pp. 765-772, 2002.

[30] A. M. Musa, E. A. G. Khalil, F. A. Mahgoub, A. M. Y. Elkadaru, A. M. El Hassan, and S. Hamad, "Efficacy of liposomal amphotericin B (AmBisome) in the treatment of persistent post-kalaazar dermal leishmaniasis (PKDL)," Annals of Tropical Medicine and Parasitology, vol. 99, no. 6, pp. 563-569, 2005.

[31] A. M. Musa, E. A. G. Khalil, A. Ismail et al., "Safety, immunogenicity and possible efficacy of immunochemotherapy of persistent post kala-azar dermal leishmaniasis (PKDL)," Sudanese Journal of Dermatology, vol. 3, pp. 62-72, 2005.

[32] S. A. Shelburne III, R. J. Hamill, M. C. Rodriguez-Barradas et al., "Immune reconstitution inflammatory syndrome: emergence of a unique syndrome during highly active antiretroviral therapy," Medicine, vol. 81, no. 3, pp. 213-227, 2002.

[33] M. A. French, "Disorders of immune reconstitution in patients with HIV infection responding to antiretroviral therapy," Current HIV/AIDS Reports, vol. 4, no. 1, pp. 16-21, 2007.

[34] T. Bicanic, G. Meintjes, K. Rebe et al., "Immune reconstitution inflammatory syndrome in HIV-associated cryptococcal meningitis: a prospective study," Journal of Acquired Immune Deficiency Syndromes, vol. 51, no. 2, pp. 130-134, 2009.

[35] J. H. Elliott, K. Vohith, S. Saramony et al., "Immunopathogenesis and diagnosis of tuberculosis and tuberculosis- associated immune reconstitution inflammatory syndrome during early antiretroviral therapy," Journal of Infectious Diseases, vol. 200, no. 11, pp. 1736-1745, 2009.

[36] J. W. T. Elston and H. Thaker, "Immune reconstitution inflammatory syndrome," International Journal of STD and AIDS, vol. 20, no. 4, pp. 221-224, 2009.

[37] M. A. French, "Immune reconstitution inflammatory syndrome: a reappraisal," Clinical Infectious Diseases, vol. 48, no. 1, pp. 101-107, 2009.

[38] H. van Tieu, J. Ananworanich, A. Avihingsanon et al., "Immunologic markers as predictors of tuberculosis-associated immune reconstitution inflammatory syndrome in HIV and tuberculosis coinfected persons in thailand," AIDS Research and Human Retroviruses, vol. 25, no. 11, pp. 1083-1089, 2009.

[39] M. A. French, N. Lenzo, M. John et al., "Immune restoration disease after the treatment of imrrmnodeficient THIVinfected patients with highly active antiretroviral therapy," $H I V$ Medicine, vol. 1, no. 2, pp. 107-115, 2000.

[40] S. A. Shelburne, M. Montes, and R. J. Hamill, "Immune reconstitution inflammatory syndrome: more answers, more questions," Journal of Antimicrobial Chemotherapy, vol. 57, no. 2, pp. 167-170, 2006.

[41] E. H. Amerson and T. A. Maurer, "Immune reconstitution inflammatory syndrome and tropical dermatoses," Dermatologic Clinics, vol. 29, no. 1, pp. 39-43, 2011.

[42] P. Blanche, B. Gombert, O. Rivoal, S. Abad, D. Salmon, and A. Brezin, "Uveitis due to Leishmania major as part of HAARTinduced immune restitution syndrome in a patient with AIDS," Clinical Infectious Diseases, vol. 34, no. 9, pp. 1279-1280, 2002.
[43] H. P. Mutimer, Y. Akatsuka, T. Manley et al., "Association between immune recovery uveitis and a diverse intraocular cytomegalovirus-specific cytotoxic T cell response," Journal of Infectious Diseases, vol. 186, no. 5, pp. 701-705, 2002.

[44] R. F. Miller, P. G. Isaacson, M. Hall-Craggs et al., "Cerebral $\mathrm{CD}^{+}$lymphocytosis in HIV-1 infected patients with immune restoration induced by HAART," Acta Neuropathologica, vol. 108, no. 1, pp. 17-23, 2004.

[45] F. Gray, C. Bazille, H. Adle-Biassette, J. Mikol, A. Moulignier, and F. Scaravilli, "Central nervous system immune reconstitution disease in acquired immunodeficiency syndrome patients receiving highly active antiretroviral treatment," Journal of NeuroVirology, vol. 11, supplement 3, pp. 16-22, 2005.

[46] O. Lortholary, A. Fontanet, N. Mémain, A. Martin, K. Sitbon, and F. Dromer, "Incidence and risk factors of immune reconstitution inflammatory syndrome complicating HIV-associated cryptococcosis in France," AIDS, vol. 19, no. 10, pp. 1043-1049, 2005.

[47] P. Phillips, S. Bonner, N. Gataric et al., "Nontuberculous mycobacterial immune reconstitution syndrome in HIVinfected patients: spectrum of disease and long-term folowup," Clinical Infectious Diseases, vol. 41, no. 10, pp. 1483-1497, 2005.

[48] G. Breton, H. Adle-Biassette, A. Therby et al., "Immune reconstitution inflammatory syndrome in HIV-infected patients with disseminated histoplasmosis," AIDS, vol. 20, no. 1, pp. 119-121, 2006.

[49] M. D. Batista, A. M. Porro, S. M. Maeda et al., "Leprosy reversal reaction as immune reconstitution inflammatory syndrome in patients with AIDS," Clinical Infectious Diseases, vol. 46, no. 6, pp. e56-e60, 2008.

[50] D. B. A. Tan, Y. K. Yong, H. Y. Tan et al., "Immunological profiles of immune restoration disease presenting as mycobacterial lymphadenitis and cryptococcal meningitis," HIV Medicine, vol. 9, no. 5, pp. 307-316, 2008.

[51] J. Tamburini, D. Grimaldi, J. D. Chiche, F. Bricaire, and P. Bossi, "Cytokine pattern in Kaposi's sarcoma associated with immune restoration disease in HIV and tuberculosis coinfected patients," AIDS, vol. 21, no. 14, pp. 1980-1983, 2007.

[52] J. F. Morlese, C. M. Orkin, R. Abbas et al., "Plasma IL-6 as a marker of mycobacterial immune restoration disease in HIV-1 infection," AIDS, vol. 17, no. 9, pp. 1411-1413, 2003.

[53] N. Seddiki, S. C. Sasson, B. Santner-Nanan et al., "Proliferation of weakly suppressive regulatory $\mathrm{CD} 4^{+} \mathrm{T}$ cells is associated with over-active $\mathrm{CD} 4^{+} \mathrm{T}$-cell responses in HIV-positive patients with mycobacterial immune restoration disease," European Journal of Immunology, vol. 39, no. 2, pp. 391-403, 2009.

[54] H. Y. Sun and N. Singh, "Immune reconstitution inflammatory syndrome in non-HIV immunocompromised patients," Current Opinion in Infectious Diseases, vol. 22, no. 4, pp. 394-402, 2009.

[55] C. M. Worsley, M. S. Suchard, W. S. Stevens, A. van Rie, and D. M. Murdoch, "Multi-analyte profiling of ten cytokines in South African HIV-infected patients with Immune Reconstitution Inflammatory Syndrome (IRIS)," AIDS Research and Therapy, vol. 7, article 36, 2010.

[56] T. Puthanakit, P. Oberdorfer, S. Punjaisee, P. Wannarit, T. Sirisanthana, and V. Sirisanthana, "Immune reconstitution syndrome due to bacillus Calmette-Guérin after initiation of antiretroviral therapy in children with HIV infection," Clinical Infectious Diseases, vol. 41, no. 7, pp. 1049-1052, 2005. 
[57] G. Matsuzaki and M. Umemura, "Interleukin-17 as an effector molecule of innate and acquired immunity against infections," Microbiology and Immunology, vol. 51, no. 12, pp. 1139-1147, 2007.

[58] T. J. Scriba, B. Kalsdorf, D. A. Abrahams et al., "Distinct, specific IL-17- and IL-22-producing $\mathrm{CD}^{+}{ }^{+} \mathrm{T}$ cell subsets contribute to the human anti-mycobacterial immune response," Journal of Immunology, vol. 180, no. 3, pp. 1962-1970, 2008.

[59] A. R. Tappuni, "Immune reconstitution inflammatory syndrome," Advances in Dental Research, vol. 23, no. 1, pp. 90-96, 2011.

[60] S. D. Lawn, L. G. Bekker, and R. F. Miller, "Immune reconstitution disease associated with mycobacterial infections in HIV-infected individuals receiving antiretrovirals," The Lancet Infectious Diseases, vol. 5, no. 6, pp. 361-373, 2005.

[61] L. R. V. Antonelli, Y. Mahnke, J. N. Hodge et al., "Elevated frequencies of highly activated $\mathrm{CD} 4^{+} \mathrm{T}$ cells in $\mathrm{HIV}^{+}$patients developing immune reconstitution inflammatory syndrome," Blood, vol. 116, no. 19, pp. 3818-3827, 2010.

[62] A. Tadesse and Z. Hurissa, "Leishmaniasis (PKDL) as a case of immune reconstitution inflammatory syndrome (IRIS) in HIVpositive patient after initiation of anti-retroviral therapy (ART)," Ethiopian Medical Journal, vol. 47, no. 1, pp. 77-79, 2009.

[63] S. Antinori, E. Longhi, G. Bestetti et al., "Post-kala-azar dermal leishmaniasis as an immune reconstitution inflammatory syndrome in a patient with acquired immune deficiency syndrome," British Journal of Dermatology, vol. 157, no. 5, pp. 10321036, 2007.

[64] E. A. G. Khalil, N. B. Ayed, A. M. Musa et al., "Dichotomy of protective cellular immune responses to human visceral leishmaniasis," Clinical and Experimental Immunology, vol. 140, no. 2, pp. 349-353, 2005. 


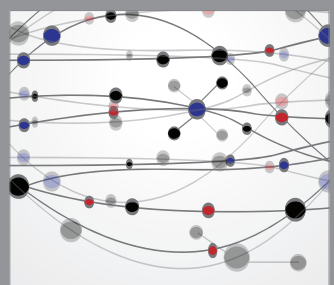

The Scientific World Journal
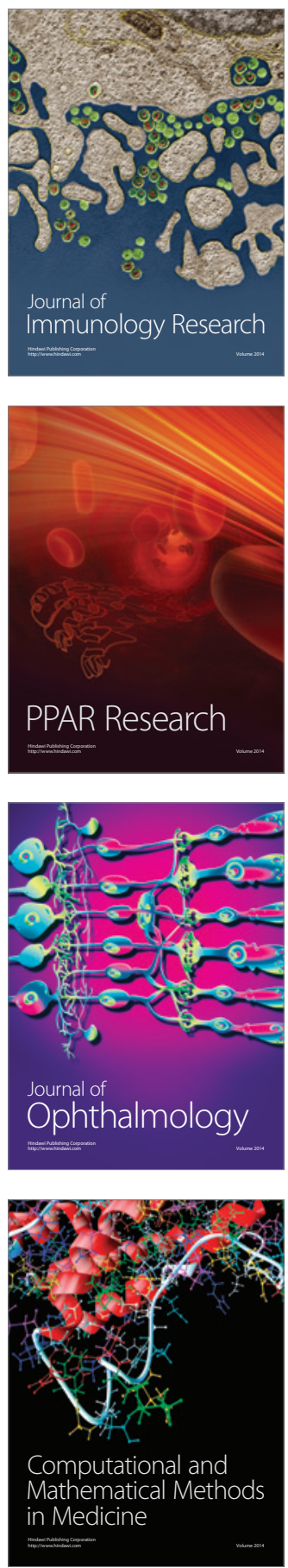

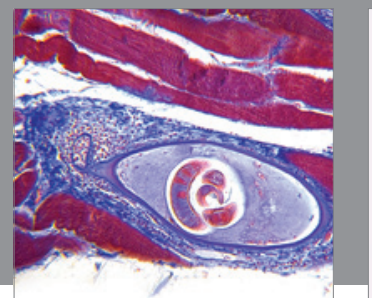

Gastroenterology

Research and Practice
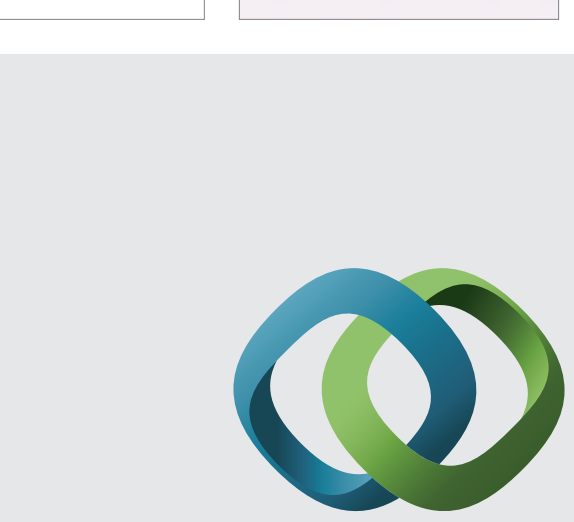

\section{Hindawi}

Submit your manuscripts at

http://www.hindawi.com
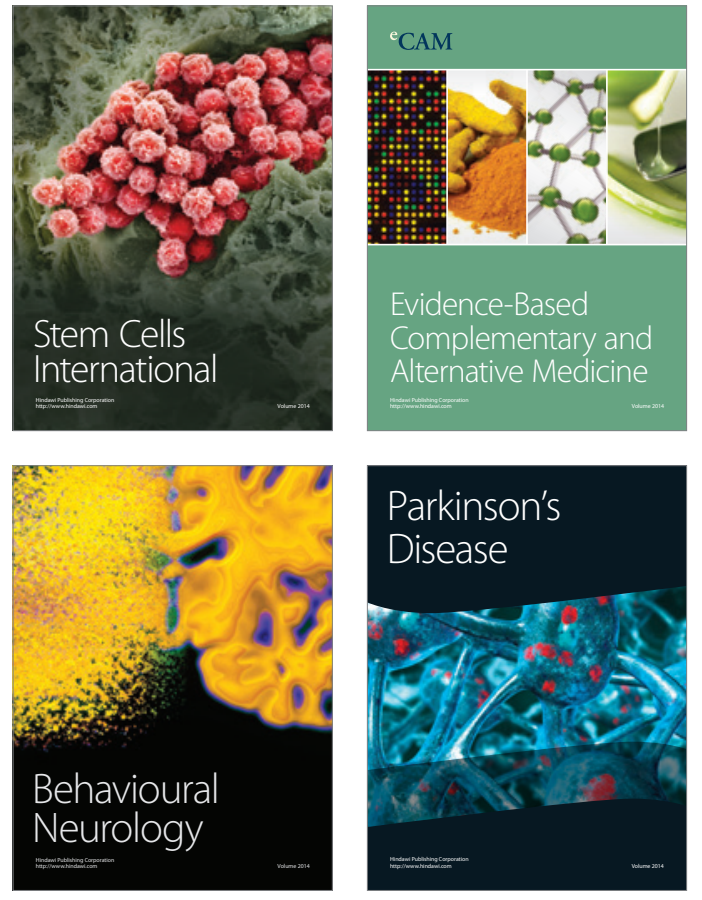
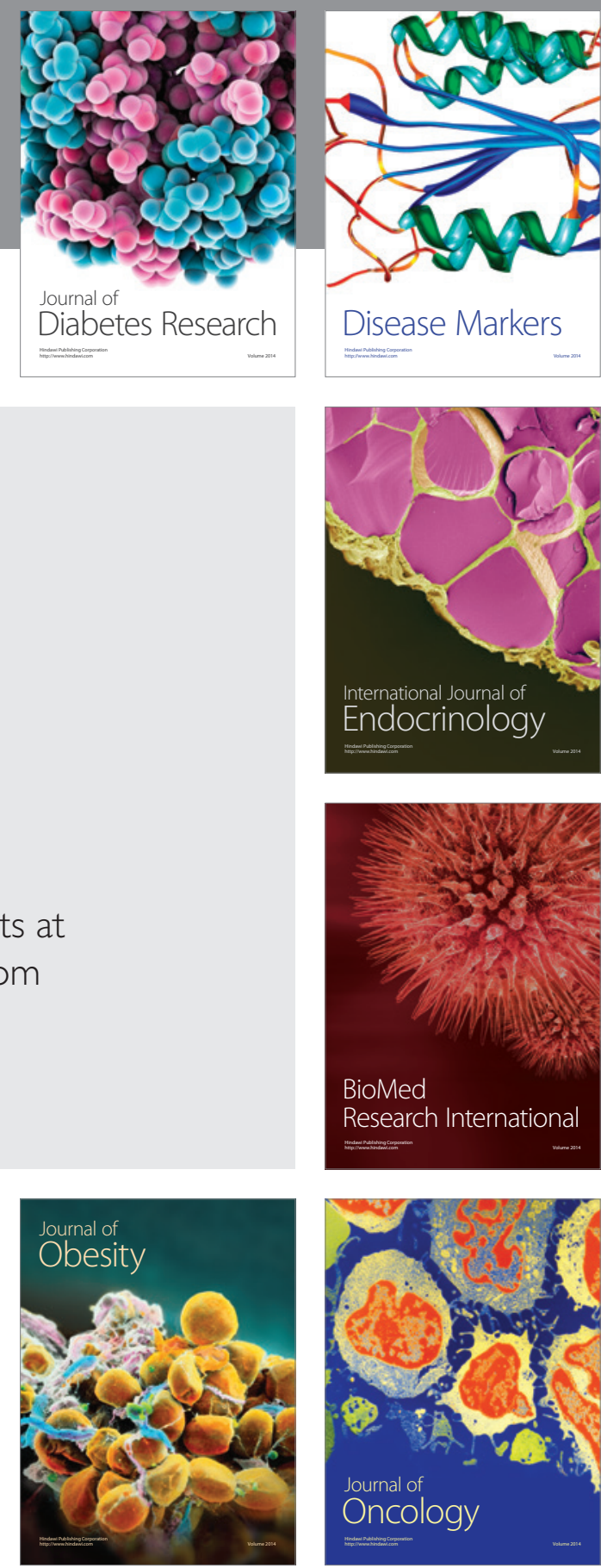

Disease Markers
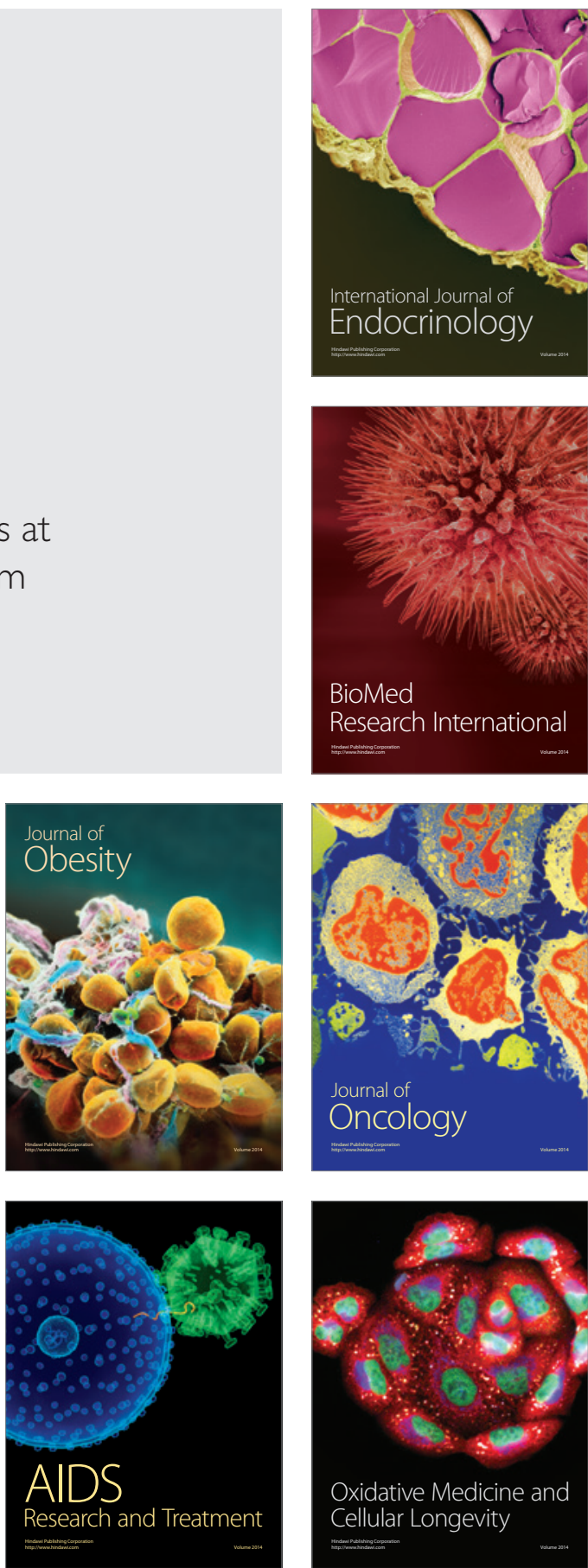